\title{
HERMITIAN OPERATORS AND ISOMETRIES ON SUMS OF BANACH SPACES
}

\author{
by R. J. FLEMING and J. E. JAMISON
}

(Received 5th January 1987)

\section{Introduction}

Let $E$ be a Banach sequence space with the property that if $\left(\alpha_{i}\right) \in E$ and $\left|\beta_{i}\right| \leqq\left|\alpha_{i}\right|$ for all $i$ then $\left(\beta_{i}\right) \in E$ and $\left\|\left(\beta_{i}\right)\right\|_{E} \leqq\left\|\left(\alpha_{i}\right)\right\|_{E}$. For example $E$ could be $c_{0}, p^{p}$ or some Orlicz sequence space. If $\left(X_{n}\right)$ is a sequence of real or complex Banach spaces, then $E$ can be used to construct a vector sequence space which we will call the $E$ sum of the $X_{n}$ 's and symbolize by $\oplus_{E} X_{n}$. Specifically, $\oplus_{E} X_{n}=\left\{\left(x_{n}\right) \mid\left(x_{n}\right) \in X_{n}\right.$ and $\left.\left(\left\|x_{n}\right\|\right) \in E\right\}$. The $E$ sum is a Banach space with norm defined by: $\left\|\left(x_{n}\right)\right\|=\left\|\left(\left\|x_{n}\right\|\right)\right\|_{E}$. This type of space has long been the source of examples and counter-examples in the geometric theory of Banach spaces. For instance, Day [7] used $E=l^{p}$ and $X_{k}=l^{q_{k}}$, with appropriate choice of $q_{k}$, to give an example of a reflexive Banach space not isomorphic to any uniformly convex Banach space. Recently VanDulst and Devalk [33] have considered Orlicz sums of Banach spaces in their studies of Kadec-Klee property.

In [8] we showed that a Banach space with a one-unconditional Schauder basis could be written as an $E$ sum of Hilbert spaces. In [9] and [14] we used the decomposition to obtain characterizations of the surjective isometries as well as the 1-parameter groups of such isometries. Simpler proofs of some of these results have been recently obtained by Arazy, see [1].

Recently Rosenthal [26], has obtained several results concerning the geometric structure of real Banach spaces which are 1-unconditional sums of Hilbert spaces of dimension at least two. To state Rosenthal's main result on isometries we need some terminology. An operator $A$ is said to be skew hermitian if $\operatorname{Re}\{f(A x)\}=0$ for all linear functionals $f$ and all vectors $x$ such that $f(x)=\|f\|\|x\|$. A real Banach space $U$ with a normalized one unconditional basis $\left(u_{i}\right)$ is said to be pure if there are no rank-two skew-Hermitian operators on $U$. We can state the main isometry result of Rosenthal:

Theorem. Let $\left(H_{a}\right)_{\alpha \in \Gamma}$ be Hilbert spaces all of dimension at least $t w o, \mathbf{u}=\left(u_{\alpha}\right)_{a \in \Gamma} a$ one-unconditional basis for a pure space $U$, and $B=\left(\sum_{\Gamma} \oplus H_{\alpha}\right)_{u}$. Let $S(B)$ denote the set of all bijections $\sigma: \Gamma \rightarrow \Gamma$ so that

(a) $\left(u_{\sigma(a)}\right)$ is isometrically equivalent to $\left(u_{a}\right)_{a \in \Gamma}$ and 
(b) $H_{\sigma(\alpha)}$ is isometric to $H_{\alpha}$.

Let $\sigma$ in $S(B)$. For each $\alpha \in \Gamma$, let $T_{\alpha}: H_{\alpha} \rightarrow H_{o(\alpha)}$ be a surjective isometry. There is a unique $T$ in $I(B)$ so that for all $x=\left(x_{\alpha}\right)_{\alpha \in \Gamma}$ in $B,(T x)_{\sigma(\alpha)}=T_{\alpha} x_{\alpha}$ for all $\alpha \in \Gamma$. Conversely, every $T$ in $I(B)$ is of this form.

This theorem obviously applies to Banach spaces over the complex field and should be compared with our Theorem 3.7. In our case, the factor spaces can be more general because we don't require them to be Hilbert spaces. For instance, the factor spaces can be sequences of Banach spaces which admit only trivial hermitian operators.

We have also been influenced by the recent paper of Jakimovski and Russell [17]. In this paper the authors' primary objective was the representation of the linear functionals on certain spaces. To accomplish this, they show how many of the spaces in ergodic theory, harmonic analysis, and summability theory are isometric to $E$-sums of appropriate sequences of Banach spaces.

For instance one of the sequence spaces which arises in summability theory is the following.

Example 0.1. $w_{p}^{0}$ is defined to be the set of all complex sequences $\left(x_{n}\right)$ for which $\lim _{N \rightarrow \infty} 1 / N \sum_{k=1}^{N}\left|x_{k}\right|^{p}=0$. For $1 \leqq p<\infty, w_{p}^{0}$ is a Banach space with norm: $\|x\|=$ $\sup \left(\left.2^{-k} \sum_{2^{k}}^{2^{k+1}-1}\left|x_{i}\right|\right|^{p}\right)^{1 / p}$. This space is isometric to $\oplus_{c 0} B_{k}$ where $B_{k}=\left\{x=\left(x_{i}\right) 2^{k} \leqq\right.$ $\left.i \leqq 2^{k+1}-1\right\}$, with coordinate wise addition and scalar multiplication. The norm on $B_{k}$ is: $\|x\|_{k}=2^{-k / p}\left\|\left(x_{i}\right)\right\|_{p}$, where \|\|$_{p}$ is the usual $l_{p}$ norm.

There is a "continuous" version of this space which was studied by Borwein in [6] and we will consider it in later section.

The Banach spaces considered in [17] may not be generally thought of as "classical" Banach spaces, but they are still interesting objects of study from the geometrical point of view. As a result of Jakimovski and Russell's work we can determine the form of the surjective linear isometries of many of the spaces considered in [17]. Our results are not limited to these spaces and the representations of the isometries for the spaces in [17] are obtained as corollaries of more general results. For example we will determine the isometries of the space $w_{p}^{0}$ by obtaining a result for $c_{0}$ sums of Banach spaces with trivial $L^{\infty}$-structure and then show that $w_{p}^{0}$ belongs to this class.

Our primary goal in this paper is to obtain representations of the isometries of certain $E$-sums of Banach spaces. Because of our techniques, there are other types of operators we consider because of their close ties with isometries. In particular, we obtain characterizations of hermitian operators for the various spaces in question. Once the structure of the hermitians is determined we describe precisely the action of the isometries on $\oplus_{E} X_{n}$ provided the "factor spaces" $X_{n}$ belong to certain classes.

In the last part of the paper we will give some results concerning hyponormal operators (see [23]) as well as adjoint abelian operators (see [32]).

\section{Definitions and terminology}

All Banach spaces considered in this paper will be linear spaces over the complex 
numbers. Given a Banach space $X, B(X)$ and $H(X)$ will denote the bounded and hermitian operators respectively. Recall that $T \in H(X)$ iff one of the following conditions hold:

(h.1) $\left\|e^{i a T}\right\|=1$ for all $\alpha \in \mathbb{R}$,

(h.2) $T$ is the generator of a uniformly continuous group of isometries,

(h.3) $\left\|e^{i a T}\right\| \leqq 1$ for $\alpha \in \mathbb{R}$,

(h.4) $\|I+i \alpha T\|=1+O(\alpha) \quad$ as $\quad \alpha \rightarrow 0$ or,

(h.5) $W(T)=\{[T x, x]\} \mid[x, x]=1\} \subseteq \mathbb{R}$, where [, ] is a semi inner product (s.i.p.) compatible with the norm on $X$.

For the most part we will use (h.5) and so we give a few properties of semi inner products. See Lumer [21] and [22] for general properties of semi inner products.

Definition 1.1. Let $X$ be a normed linear space. $A$ s.i.p. is a function [, ]: $X \times X \rightarrow C$ such that

(1.1.1) $[\alpha x+y, z]=\alpha[x, z]+[y, z]$ for $x, y, z$ in $X$ and $\alpha$ in $C$,

(1.1.2) $[x, x] \geqq 0$ and equality holds only if $x=\theta$.

(1.1.3) $|[x, y]| \leqq[x, x]^{1 / 2}[y, y]^{1 / 2}$

The s.i.p. is said to be compatible with the norm on $X$ if

(1.1.4) $\|x\|^{2}=[x, x]$ for every $x \in X$.

Remarks. It is well known that every normed linear space has at least one s.i.p. compatible with the norm. Furthermore, a space has unique s.i.p. iff the unit sphere is smooth. A s.i.p. is symmetric iff the induced norm is Euclidean. A s.i.p. need not be conjugate homogenous in the second argument but there always exists such a s.i.p. compatible with the norm. We will assume that our s.i.p.'s have this property. Finally, we will use the fact ([21]) that any compatible s.i.p. can be used to test (h.5).

Now we suppose that $E$ is a Banach sequence space satisfying the conditions outlined in the introduction and furthermore we will assume that the natural basis is a normalized 1-unconditional (or hyperorthogonal) Schauder basis for $E$. Thus if $X=$ $\oplus_{E} X_{n}$, the sequence $\left(X_{n}\right)$ is an unconditional Schauder decomposition of $X$ and so each $x \in X$ can be written uniquely as $x=\sum x_{j}$, where $x_{j} \in X_{j}$. For each $k$ we let $P_{k} ; X \rightarrow X_{k}$ be the projection $P_{k}\left(\sum x_{j}\right)=x_{k}$ and $Q_{k} X_{k} \rightarrow X$ be the injection $Q_{k} x_{k}=\sum y_{i}$ where $y_{i}=0$ for $\mathrm{i} \neq k$, and $y_{k}=x_{k}$. With each $A \in B(X)$ we associate an operator matrix $\left(A_{j k}\right)$ where $A_{j k}=P_{j} A Q_{k}$. Since we are dealing with an unconditional decomposition, the usual matrix operations apply.

\section{A special case- $\oplus_{\mathrm{c}_{0}} X_{\mathbf{n}}$}

We begin by considering $c_{0}$ sums of Banach spaces. This situation is the most transparent and should serve as an introduction to our methods. In what follows we use 
$\|\cdot\|$ to denote the norm on each $X_{n}$ and the norm on $X=\oplus_{c_{0}} X_{n}$ will be given by $\|x\|_{\infty}=\max \left\|x_{k}\right\|$. Occasionally, we use \|\|$_{n}$ to denote the norm on $X_{n}$ if it is important to make a distinction.

Since for each $x=\left(x_{n}\right) \in X$ there exists at least one positive integer $k=k(x)$ for which $\|x\|_{\infty}=\left\|x_{k}\right\|$, it is easy to give a compatible s.i.p. for $X$.

Lemma 2.1. For each $x \in X$ let $k(x)$ be the least positive integer for which $\left\|x_{k}\right\|=\|x\|_{\infty}$. Let $[,]_{k}$ denote any compatible s.i.p. on $X_{k}$. For each $x$ and $y$ let

$$
[x, y]:=\left[x_{k(y)}, y_{k(y)}\right]_{k(y)} .
$$

Then $[$,$] is a s.i.p. on X$ compatible with the norm.

The proof is routine and we omit it.

Proposition 2.2. $T$ is a hermitian operator on $\oplus_{c_{0}} X_{n}$ iff there exists a bounded sequence $\left(T_{k}\right)$ of operators such that $T_{k} \in H\left(X_{k}\right)$ for each $k$ and $T\left(x_{n}\right)=\left(T_{n} x_{n}\right)$.

Proof. These conditions are clearly sufficient. To see that they are necessary let $T_{i j}=P_{i} T Q_{j}$. Let $k \neq j$ and choose $x_{k} \in X_{k}, x_{j} \in X_{j}$ such that $\left\|x_{k}\right\| \geqq\left\|x_{j}\right\| . \quad x=$ $\left(0, \ldots, x_{k}, 0, \ldots, x_{j}, 0 ..\right) \in \oplus_{c_{0}} X_{n}$ and using notation of Lemma $(2.1), k(x)=k . \quad T x=$ $\left(T_{i k} x_{k}+T_{i j} x_{j}\right)$. From (2.1.1) we have

$$
[T x, x]=\left[T_{k k} x_{k}, x_{k}\right]_{k}+\left[T_{k j} x_{j}, x_{k}\right]_{k} .
$$

This has to be real for all choices of $x_{k}$ and $x_{j}$ for which $\left\|x_{k}\right\| \geqq\left\|x_{j}\right\|$. First choose $x_{j}=\theta_{j}$ to get that $\left[T_{k k} x_{k}, x_{k}\right]_{k} \in \mathbb{R}$ for all $x_{k}$. Now replace $x_{j}$ by $i x_{j}$ to get

$$
i\left[T_{k j} x_{j}, x_{k}\right]_{k} \in \mathbb{R} \text { for all admissible } x_{k}, x_{j} .
$$

It is clear that

$$
\left[T_{k j} x_{j}, x_{k}\right]_{k}=0 \text { for all admissible } x_{k}, x_{j} \text {. }
$$

Now let $z_{k}, z_{j}$ be non zero vectors in $X_{k}, X_{j}$ respectively. Since $z_{k} /\left\|z_{k}\right\|$ and $z_{j} /\left\|z_{j}\right\|$ are admissible it follows that

$$
\left[T_{k j} \frac{z_{j}}{\left\|z_{j}\right\|}, \frac{z_{k}}{\left\|z_{k}\right\|}\right]=0
$$

From this last equality it follows that

$$
\left\|T_{k j} z_{j}\right\|^{2}=0
$$


for all $z_{j}$ and so $T_{k j}=0$. The fact that the diagonal sequence must be bounded follows from the boundedness of $T$.

This proposition will allow us to prove a theorem on the isometries of $\oplus_{c_{0}} X_{n}$. Before stating the theorem there are several notions which will arise as hypotheses about the factor spaces $X_{n}$ which we must consider.

Let $v$ be a normalized absolute norm on $\mathbb{R}^{2}$. That is, $v$ is a norm with $v\left(x_{1}, x_{2}\right)=$ $v\left(\left|x_{1}\right|,\left|x_{2}\right|\right)$ and $v(1,0)=v(0,1)=1$. Paya Albert [25] introduced the notion of a " $v$-projection" which generalized the earlier idea of an $L^{p}$-projection due to Behrends [3]. A bounded projection $Q$ on a Banach space $X$ is a $v$ projection if for every $x$ $\|x\|=v(\|Q x\|,\|x-Q x\|)$. In the $L^{p}$ case, $v\left(x_{1}, x_{2}\right)=\left(\left|x_{1}\right|^{p}+\left|x_{2}\right|^{p}\right)^{1 / p}$ for $1<p<\infty$, while $v\left(x_{1}, x_{2}\right)=\operatorname{Max}\left(|x|,\left|x_{2}\right|\right)$ for $p=\infty$. $X$ has trivial $L^{p}$ structure if 0 and $I$ are the only $L^{p}$ projections. For the purposes of this section, $L^{\infty}$ projections will suffice but we will state all of the lemmas for the general case.

Lemma 2.3. Let $X$ and $Y$ be Banach spaces. Let $U$ be a surjective isometry from $X$ to $Y$. Let $T$ be hermitian on $X$ and $Q$ be a v projection on $X$. Then,

$$
\begin{aligned}
& Q \text { is hermitian on } X, \\
& U Q U^{-1} \text { is a v projection on } Y, \text { and } \\
& U T U^{-1} \text { is hermitian on } Y .
\end{aligned}
$$

Proof. First we prove (2.3.a). Since $Q^{2}=Q$ it follows that for any $\alpha \in \mathbb{R}, e^{i \alpha Q}=$ $(I-Q)+e^{i \alpha} Q$. Hence, $Q e^{i \alpha Q}=e^{i \alpha} Q$ and $(I-Q) e^{i \alpha Q}=(I-Q)$. Now for any $x \in X$,

$$
\begin{aligned}
\left\|e^{i \alpha Q} x\right\| & =v\left(\left\|Q e^{i \alpha Q} x\right\|,\left\|(I-Q) e^{i \alpha Q} x\right\|\right) \\
& =v\left(\left\|e^{i \alpha} Q x\right\|,\|(I-Q) x\|\right) \\
& =\|x\| .
\end{aligned}
$$

This last equality follows from the fact that $Q$ is a $v$-projection. From (h.1) it is clear that $Q$ is hermitian.

To prove (2.3.b) let $y \in Y$. Then 


$$
\left\|U Q U^{-1} y\right\|=\left\|Q U^{-1} y\right\| \text { and likewise }\left\|y-U Q U^{-1} y\right\|=\left\|U^{-1} y-Q U^{-1} y\right\| .
$$

Thus,

$$
\begin{aligned}
v\left(\left\|U Q U^{-1} y\right\|,\left\|y-U Q U^{-1} y\right\|\right) & =v\left(\left\|Q U^{-1} y\right\|,\left\|U^{-1} y-Q U^{-1} y\right\|\right) \\
& =\left\|U y^{-1}\right\|=\|y\| .
\end{aligned}
$$

The proof of (2.3.c) follows from the equality

$$
\exp \left(i \alpha U T U^{-1}\right)=U e^{i \alpha T} U^{-1}
$$

and the fact that $U$ is a surjective isometry.

Remarks. (2.3.c) can be used to give a short proof of the classical Banach Stone Theorem for isometries between $C\left(\Omega_{1}\right)$ and $C\left(\Omega_{2}\right)$, where $\Omega_{1}$ and $\Omega_{2}$ are compact Hausdorf spaces. See Section 6.

Before stating our first result on isometries we need an additional fact concerning $v$-projections.

Lemma 2.4. Let $Q: \oplus_{E} X_{n} \rightarrow \oplus_{E} X_{n}$ be the "diagonal map" $Q\left(x_{n}\right)=\left(Q_{n n} x_{n}\right)$. If $Q$ is a $v$-projection, then each $Q_{n n}$ is also a v-projection on $X_{n}$.

Proof. Suppose $Q$ is a $v$-projection. For each positive integer $k, Q_{k k}$ is clearly a projection and furthermore,

$$
\left\|\left(0,0, \ldots, \mathrm{x}_{k}, 0 \ldots\right)\right\|_{E}=v\left(\left\|\left(0, \ldots, Q_{k k} x_{k}, 0 \ldots\right)\right\|_{E},\left\|\left(0, \ldots, x_{k}-Q_{k k} x_{k}, 0\right)\right\|_{E}\right.
$$

i.e. $\left\|x_{k}\right\|_{k}=v\left(\left\|Q_{k k} x_{k}\right\|_{k},\left\|x_{k}-Q_{k k} x_{k}\right\|_{k}\right)$. Thus, $Q_{k}$ is a $v$ projection on $X_{k}$.

Theorem 2.5. Let $\left(X_{n}\right)$ be a sequence of complex Banach spaces such that any one of the following holds for every $X_{n}$.

(2.5a) $\quad X_{n}$ has trivial $L^{\infty}$-structure.

(2.5b) $X_{n}$ has only trivial hermitian projections.

(2.5c) $X_{n}$ has only trivial hermitian operators.

(2.5d) $\quad H\left(X_{n}\right)^{\prime}$ contains no nontrivial hermitian projections.

(2.5e) $A H\left(X_{n}\right) B=\{0\}$ implies that $A=0$ or $B=0$.

$T$ is a surjective isometry of $\oplus_{c_{0}} X_{n}$ iff there exists a permutation $\pi$ of the positive 
integers $Z^{+}$and a sequence of isometric operators $U_{n \pi(n)}$ such that $T\left(x_{n}\right)=U_{n \pi(n)} x_{\pi(n)}$ for each $\left(x_{n}\right) \in \oplus_{c 0} X_{n}$. Moreover, the space $X_{\pi(n)} \cong X_{n}$.

Proof. Let $T$ be a surjective isometry of $\oplus_{c_{0}} x_{n}$ and suppose that the matrix representations of $T$ and $T^{-1}$ are denoted by $\left(T_{i j}\right)$ and $\left(S_{i j}\right)$ respectively. For each positive integer $k$ let $Q^{k}$ be defined by

$$
Q^{k}(x)=\left(0,0, \ldots, x_{k}, 0,0 \ldots\right) .
$$

From Lemma (2.3), $T^{-1} Q^{k} T$ and $T Q^{k} T^{-1}$ are both hermitian and $L^{\infty}$ projections. By Proposition (2.2) $T^{-1} Q^{k} T$ is diagonal and by Lemma 2.4 each diagonal entry is both hermitian and an $L^{\infty}$ projection on the corresponding factor space. Hence, if (2.5a), (2.5b) $(2.5 \mathrm{c})$ holds, these diagonal operators must be either the zero operator or the identity.

If (2.5d) holds we obtain a similar conclusion as follows: since the hermitians on $\oplus_{c_{0}} X_{n}$ are diagonal, $Q^{k}$ commutes with every hermitian. As a consequence, $T^{-1} Q^{k} T$ is a diagonal operator with hermitian projections on the diagonal. It follows that each diagonal entry of $T^{-1} Q^{k} T$ must commute with every hermitian operator on the corresponding factor space. By (2.5d), the only such projections are 0 or $I$.

Therefore for any of the first four hypotheses we get that for each positive integer $k$

$$
\begin{gathered}
S_{i k} T_{k j}=0_{i j} \text { for } i \neq j \text { (where } 0_{i j} \text { is the zero operator) } \\
I_{j} \text { or } 0_{j} \text { if } i=j .
\end{gathered}
$$

Since $T^{-1} Q^{k} T$ is not the zero operator, there must exist at least one positive integer which we denote by $\pi(k)$ for which

$$
S_{\pi(k) k} T_{k \pi(k)}=I_{\pi(k)}
$$

Since,

$$
\left[x_{n}, x_{n}\right]=\left[\left(T^{-1} T\right)_{n n} x_{n}, x_{n}\right]=\sum\left[S_{n k} T_{k n} x_{n}, x_{n}\right],
$$

it follows that given $k$ there is at most one $\pi(k)$ for which (2.5.3) holds.

Therefore, the mapping $k \rightarrow \pi(k)$ is a one to one function on $\mathbb{Z}^{+}$. Moreover, the map $k \rightarrow \pi(k)$ is surjective, for if $\lambda \notin \pi\left(\mathbb{Z}^{+}\right), T z=0$ when $z=\left(0, \ldots, x_{\lambda}, 0 \ldots\right)$ and this is impossible. We say that $\pi$ is induced by the conjugation $Q \rightarrow T^{-1} Q T$.

Since $T\left(T^{-1} Q^{k} T\right) T^{-1}=Q^{k}$ for each $k$, it follows that the "conjugation" $T Q^{k} T^{-1}$ induces the permutation $\sigma$ which is inverse to $\pi$. Thus as before

$$
\left(T Q^{k} T^{-1}\right)_{i j}=T_{i k} S_{k j}= \begin{cases}0_{i j} & \text { if } \quad i \neq j \\ 0_{i} & \text { if } \quad i=j \neq \sigma(k) \\ l_{\sigma(k)} & \text { if } \quad i=j=\sigma(k)\end{cases}
$$




$$
I_{\sigma(k)} \quad \text { if } \quad i=j=\sigma(k)
$$

From (2.5.3) we have

and consequently

$$
S_{\pi(k) k} T_{k j}=0_{\pi(k) j} \text { for } j \neq \pi(k)
$$

$$
T_{k \pi(k)} S_{\pi(k) k} T_{k j}=T_{k \pi(k)} 0_{\pi(k) j}=0_{k j}
$$

From (2.5.5) we have that $T_{k \pi(k)} S_{\pi(k) k}=I_{k}$ and so,

$$
T_{k j}=0_{k j} \text { for } j \neq \pi(k) .
$$

Therefore, we have shown that $\left(T_{i j}\right)$ has exactly one non zero operator entry per row and column and so for each $x=\left(x_{n}\right)$ in $\oplus_{c_{0}} X_{n}$ we have the formula

$$
(T x)_{n}=T_{n \pi(n)} x_{\pi(n)}
$$

By taking vectors with "single entries" it follows that $\left\|T_{n \pi(n)} x_{n(n)}\right\|_{n}=\left\|x_{\pi(n)}\right\|$ for each $n$. Since $T$ is surjective it follows that $T_{n \pi(n)}$ maps $X_{n(n)}$ onto $X_{n}$ and so $X_{n(n)}$ and $X_{n}$ are congruent. This essentially completes the proof of the theorem for the first four hypotheses. The proof in the case of the last hypothesis is almost exactly the same as the case that the $X_{k}$ are all Hilbert spaces and this argument is given in [9] and we will not repeat the arguments here.

Remark. See the remarks following Theorem 3.1 concerning hypotheses (2.5e).

As an illustration of this theorem we will consider several spaces from the paper of Jakimovski and Russell. The first space is $w_{p}^{0}$ which has already been defined. The second space is due to Borwein [6] and is defined as follows:

$$
W_{p}^{0}=\left\{f \mid f \text { is measurable on }[1, \infty], \lim _{T \rightarrow \infty} \frac{1}{T} \int_{1}^{T}|f|^{p}=0\right\}
$$

with the usual vector addition and scalar multiplication. As noted by Jakimovski and Russell, if one defines

$$
\left(B_{k},\|\|_{B}\right)=L^{p}\left[2^{k}, 2^{k+1}\right), \quad\|f\|_{B}=\left(2^{-k} \int_{2^{k}}^{2^{k+1}}|f|^{p}\right)^{1 / p}
$$

for $k \in \mathbb{Z}^{+}$, then $W_{p}^{0}=\bigoplus_{c_{0}} B_{k} .\left(L^{p}\left[2^{k} \cdot 2^{k+1}\right)\right.$ denotes the usual $L^{p}$ space defined by Lebesque measure on $\left[2^{k} \cdot 2^{k+1}\right)$ ).

Corollary 2.6. If $U$ is a surjective isometry of $w_{p}^{0}$ or $W_{p}^{0}$ then there exists a sequence $\left(U_{n \pi(n)}\right)$ of operators such that 
(a) for $w_{p}^{0} \pi(k)=k$ for each $k$, and there is a surjection $\sigma_{k}$ of $\left\{2^{k}, 2^{k}+1, \ldots, 2^{k+1}-1\right\}$ and a sequence of real numbers $\theta_{k}$ such that $U_{k}\left(x_{j}\right)=\left(e^{i \theta_{k}} x_{\sigma_{k}(U)}\right)$ for $2^{k} \leqq j<2^{k+1}$.

(b) for $W_{p}^{0}$, there exists for each $n$ a measurable point mapping $T_{n}$ of $J_{n}$ onto $J_{\pi(n)}$ such that if $f_{n}=f I_{J_{n}}$ then

$$
U_{n \pi(n)} f_{\pi(n)}=h_{n} f_{\pi(n)} \circ T_{n}
$$

(We have used $I_{A}$ to denote the indicator function of the set $A$ and $h_{n}$ to denote the "( $1 / p)$ th" root of the Radon-Nikodym derivative of the measure $m T^{-1}$ with respect to $m)$.

Proof. All that needs to be verified is that the "factor spaces" in each case have trivial $L^{\infty}$-structure. This fact follows from the work of Behrends [3]. He showed that $L^{p}(\mu)$ spaces have only $L^{p}$ projections and so with $1 \leqq p<\infty, L^{p}\left[2^{n}, 2^{n+1}\right)$ and $l^{p}$ have only trivial $L^{\infty}$ projections. The description of the isometries on the factor spaces is known and follows from the work of Banach and Lamperti $[2,20]$.

Remark. There are other spaces described in [17] satisfying the hypotheses of Theorem 2.5. Rather than give explicit descriptions of their isometries we choose to point out which of the hypotheses is satisfied in each case. For example, the space $0[A]_{p}$ is isometric to a $c_{0}$ sum weighted $l^{p}$ spaces (see page 283 of [17]). Since these $l^{p}$ spaces have trivial $L^{\infty}$ structure it follows that condition (2.5a) holds. Likewise, the space $C_{p, x}$, $p>1$, (see page 283 of [17]) is isometric to a $c_{0}$ sum of $L^{p}$ spaces and these spaces also have trivial $L^{\infty}$ structure.

So far, only one hypothesis of Theorem 2.5 has been used in our illustration of the theorem. To see an application of some of the other hypotheses, we turn to a class of Banach spaces that comes from abstract harmonic analysis.

In [19], J. LaDuke introduced a class of Banach spaces which he called $\mathscr{E}_{p}$ spaces. One of these spaces can be described as a $c_{0}$ sum. To be specific, let $I$ be a countable index set and suppose that for each $i \in I, H_{i}$ is a finite dimensional Hilbert space. $\mathscr{E}_{0}(I)$ is the * sub algebra of $\Pi_{i \in I} B\left(H_{i}\right)$ with coordinate wise operations and satisfying the condition that $\left(E_{j}\right) \in \mathscr{E}_{0}(I)$ if and only if $\left\{i \in I:\left\|E_{i}\right\| \geqq \varepsilon\right\}$ is finite for all $\varepsilon>0$. The norm for $\mathscr{E}_{0}(I)$ is sup norm, i.e. $\left\|\left(E_{i}\right)\right\|_{\infty}=\sup \left\|E_{i}\right\|$, where $\left\|E_{i}\right\|$ is the operator norm on $B\left(H_{i}\right)$.

In order to apply Theorem 2.5 to the space $\mathscr{E}_{0}(I)$ we need to show which of the hypotheses is satisfied. The lemma which follows will provide the answer. We state it in its most general form.

Lemma 2.7. Let $Z \neq C_{2}(H)$ be $B(H)$, or any minimal normed ideal in $B(H)$ in which the finite rank operators are dense. If $Q$ is a hermitian projection on $Z$ which commutes with every hermitian operator on $Z$ then $Q$ is 0 or $I$.

Proof. Let $Q$ be an hermitian projection. It follows from work of Sinclair [29], and Sourour [31] that there exists operators $A_{1}, A_{2}$ such that each $A_{i}$ is hermitian on the 
Hilbert space $H$ and for each $T \in Z, Q(T)=A_{1} T+T A_{2}$. Since $Q$ is a projection, it follows from Sourour [31] that $Q(T)=A_{1} T$ for all $T$ or else $Q(T)=T A_{2}$ for all $T$. It is obvious that in either case $A_{i}$ must be a projection on $H$. If we now envoke the hypothesis that $Q$ commutes with every hermitian on $\mathbb{Z}$ it follows that $A_{1}$ (or respectively $A_{2}$ ) must commute with every hermitian on $H$ and consequently with every operator on $H$. Clearly then $A_{1}=0_{H}$ or $I_{H}$ and so $Q$ is $0_{Z}$ or $I_{Z}$.

From the preceding lemma, it is clear that the spaces of LaDuke satisfy (2.5d). Theorem 2.5 and Sinclair's characterization of the surjective isometries of $B^{*}$ algebras yield the following result.

Corollary 2.8. Let $W$ be a surjective isometry of $\mathscr{E}_{0}(I)$. Then there exists a permutation $\phi$ of the positive integers and unitary operators $U_{i \phi(i)}, V_{i \phi(i)}$ such that for $E=\left(E_{i}\right)$,

$$
W(E)=\left\{\begin{array}{c}
U_{i \phi(i)} E_{\phi(i)} V_{\phi(i) i} \\
o r \\
U_{i \phi(i)} E_{\phi(i)}^{t} V_{\phi(i) i}
\end{array}\right.
$$

where for each $i, H_{i} \cong H_{\phi(i)}$ and " $t$ " denotes the transpose with respect to some orthonormal basis for $H_{\phi(i)}$.

\section{Hermitians and isometries on $\oplus_{E} X_{n}$}

In this section we will investigate the structure of the hermitian operators and the surjective isometries of more general sums. Much of what is done in this section is generalization of results from [8] and [9]. We begin all of this by recalling essential notions from [8].

Definition 3.1. A s.i.p. on $X=\oplus_{E} X_{n}$ is said to be sufficiently $l^{p}$-like if there exists a sequence of nonnegative functions $a_{i}$ defined on $X$ such that

(3.1.3) For every $(i, j) \exists x_{i} \in X_{i}, x_{j} \in X_{j}$ such that if $x=x_{i}+x_{j}$, then it follows that $a_{i}(x)=a_{j}(x)$.

(3.1.4) For every pair $k \neq j$ there exists $x \in X$ with $x_{k} \neq \theta_{k}$ and $x_{j} \neq \theta_{j}$ such that $a_{k}(x) \neq a_{j}(x)$. Let $[,]_{i}$ denote a compatible s.i.p. on $X_{i}$. For $x=\left(x_{i}\right)$ and $y=\left(y_{j}\right)$ in $X$,

(3.1.5) $[x, y]=\sum a_{i}(x)\left[x_{i}, y_{i}\right]_{i}$ defines a s.i.p. on $X$ compatible with the norm.

In [8] we showed that every Banach space with a normalized one unconditional basis $\left(e_{i}\right)$ can be written as an $E$-sum for an appropriate $E$ and possesses a sufficiently $l^{p}$-like s.i.p. In that situation, each $X_{i}$ is an $l^{2}$ space of appropriate dimension and $[,]_{i}$ is in 
fact an inner product for each $i$. We also defined two "coordinates" $i$ and $j$ to be equivalent if whenever $x=\sum x_{k} e_{k}$ and $y=\sum y_{k} e_{k}$ belong to $X$ with $\left|x_{i}\right|^{2}+\left|x_{j}\right|^{2}=$ $\left|y_{i}\right|^{2}+\left|y_{j}\right|^{2}$ and $\left|x_{n}\right|=\left|y_{n}\right|$ for $n \neq i, j$ then $\|x\|=\|y\|$. In what follows we will require that the sequence space $E$ have no equivalent coordinates. In this situation the $x_{i}$ are just complex numbers and so $\left[x_{i}, y_{i}\right]_{i}=x_{i} \bar{y}_{i}$, where $\bar{y}_{i}$ denote the complex conjugate of $y_{i}$.

We now record a lemma the proof of which is essentially contained in Lemma 4.6 of [8].

Lemma 3.2. Let $X=\oplus_{E} X_{n}$ and suppose $E$ has a 1-unconditional basis and no equivalent coordinates. Then $X$ has a sufficiently $l^{p}$ like s.i.p.

Proof. Let $[\alpha, \beta]=\sum \alpha_{k} \beta_{k} a_{k}(\beta)$ denote a s.i.p. which is sufficiently $\lambda^{p}$ like. For $x=\left(x_{n}\right)$ and $y=\left(y_{n}\right) \in X$ define

$$
[x, y]=\sum\left[x_{n}, y_{n}\right]_{n} A_{n}(x),
$$

where $A_{k}(x):=a_{k}\left(\left\|x_{k}\right\|_{k}\right)$, and $[,]_{n}$ is any compatible s.i.p. on $X_{n}$.

It follows that this is the required s.i.p. on $X$ which has the required properties and in particular (3.1.4) follows from the fact that $E$ has no equivalent coordinates.

Once the lemma is known the next theorem follows exactly as in [8]. A careful reading of the proof of Theorem 2.6 of [8] shows that only s.i.p. properties were used and consequently the argument there transfers to this situation with only minor changes.

Theorem 3.3. Let $X=\oplus_{E} X_{n}$ where $E$ has a 1-unconditional basis and no equivalent coordinates. If $T$ is hermitian on $X$ then, there exists a sequence $\left(T_{n}\right)$ of operators such that $T_{n}$ is hermitian on $X_{n}$ and $T\left(x_{k}\right)=\left(T_{k} x_{k}\right)$ for each $\left(x_{k}\right) \in X$. Furthermore the sequence $\left\{\left\|T_{n}\right\|\right\}$ is bounded. These conditions are also sufficient.

Remark. The hypothesis concerning no equivalent coordinates in $E$ rules out $l^{2}$ sums. It appears that $l^{2}$ sums are much more complicated to deal with. For instance, it is not clear that hermitians on $\dot{X}_{1} \oplus_{2} X_{2}$ need be diagonal when $X_{1}, X_{2}$ are non Hilbert spaces. See the papers of Berkson and Sourour [5] and Partington [24] for some results on this question. In [5] Berkson and Sourour showed that the hermitian operators on $l^{p}$ sums of Banach spaces are "diagonal" for $1 \leqq p<\infty, p \neq 2$. The following corollary generalizes their results to the case of Orlicz sums of Banach spaces.

Let $\phi$ be an Orlicz function satisfying the strong $\Delta_{2}$ property and further suppose that $\phi$ is differentiable and that $t^{-1} \phi^{\prime}(t)$ is one to one. If $\left(X_{n}\right)$ is a sequence of complex Banach spaces let $l_{\phi}\left(X_{n}\right)=\oplus_{E} X_{n}$, where $E$ is usual Orlicz sequence space $l_{\phi}$. It is well known that the usual basis vector $\left(\delta_{i j}\right)_{j=1}^{\infty}$ are an unconditional basis for $l_{\phi}$ when $\phi$ satisfies the strong $\Delta_{2}$ condition and furthermore this basis is 1-unconditional when $l_{\phi}$ is normed by the Luxemburg norm 


$$
\left\|\left(\alpha_{n}\right)\right\|=\inf \left\{\lambda \mid \sum \phi\left(\frac{\left|\alpha_{n}\right|}{\lambda}\right) \leqq 1\right\}
$$

The hypothesis concerning $t^{-1} \phi^{\prime}(t)$ insures (see [9] that $l_{\phi}$ has no equivalent coordinates. Thus, by Theorem 3.3 we have

Corollary 3.5. Let $E=l_{\phi}$ where $\phi$ is differentiable, satisfies the strong $\Delta_{2}$ property and $t^{-1} \phi^{\prime}(t)$ is one to one. If $\left(X_{n}\right)$ is a sequence of complex Banach spaces and $W=\oplus_{E} X_{n}$, then $T$ is hermitian on $W$ iff there is a sequence $\left(T_{n}\right)$ of uniformly bounded operators such that

$$
T\left(x_{n}\right)=\left(T_{n} x_{n}\right) \quad \text { for } \quad\left(x_{n}\right) \in W \quad \text { and } \quad T_{n} \in H\left(X_{n}\right)
$$

for every $n$.

Remark. If $\phi(t)=t^{p} / p$, then $E=l^{p}$ and we get the result of Berkson and Sourour. Also note that the hypotheses rule out $p=2$.

As noted earlier, spaces such as $E=l_{\phi}$ have at least one semi inner product which is compatible with the norm and sufficiently $l^{p}$ like. The following proposition gives such a s.i.p.

Proposition 3.6. Let $\phi$ and $\left(X_{n}\right)$ be as above. Then,

$$
\left[\left(x_{n}\right),\left(y_{n}\right)\right]=\sum_{k=1}\left[x_{k}, y_{k}\right]_{k} a_{k}(y),
$$

where

$$
a_{k}(y)=\left[\sum\left\|y_{m}\right\|_{m} \phi^{\prime}\left(\frac{\left\|y_{m}\right\|_{m}}{\|y\|}\right)\right]^{-1} \phi^{\prime}\left(\frac{\left\|y_{k}\right\|}{\|y\|}\right)
$$

is a s.i.p. compatible with the norm on $\oplus_{E} X_{n}, E=l_{\phi}$.

Proof. The fact that (3.6.1) is a compatible s.i.p. is given in [18]. It is routine to verify that it is sufficiently $l^{p}$ like.

As we noted earlier, our main quest is the characterization of isometries of $E$ sums of Banach spaces. We now have the necessary preliminaries and we proceed to the main result. Before stating the theorem we list some terminology. For the most part we are just restating conditions we have imposed on the sequence spaces $E$. Let us say that the sequence space $E$ is "admissible" if:

(a) $\left(\alpha_{i}\right) \in E$ and $\left|\beta_{i}\right| \leqq\left|\alpha_{i}\right|$ for all $i$ implies $\left(\beta_{i}\right) \in E$ and $\left\|\left(\beta_{i}\right)\right\|_{E} \leqq\left\|\left(\alpha_{i}\right)\right\|_{E}$. 
(b) the vectors $(1,0,0, \ldots),(0,1,0, \ldots)$, etc., form a 1 -unconditional (hyperorthogonal) Schauder basis for $E$.

(c) $E$ has no equivalent coordinates.

Definition 3.7. Let $E$ be an admissible sequence space. A permutation $\pi$ is said to be a symmetry of the norm if $\left\|\left(\alpha_{\pi(n)}\right)\right\|_{E}=\left\|\left(\alpha_{n}\right)\right\|_{E}$ for all $\left(\alpha_{n}\right) \in E$.

Theorem 3.7. Let $E$ be an admissible sequence space and let $\left(X_{n}\right)$ be a sequence of complex Banach spaces such that any one of the following holds for every $X_{n}$.

(3.7a) $X_{n}$ has only trivial hermitian projections.

(3.7b) $X_{n}$ has only trivial hermitian operators.

(3.7c) $\quad H\left(X_{n}\right)^{\prime}$ contains no nontrivial hermitian projections.

(3.7d) $A H\left(X_{n}\right) B=\{0\}$ implies that $A$ or $B=0$.

If $T$ is a surjective isometry of $X=\oplus_{E} X_{n}$ then there exists a symmetry $\pi$ of the sequence norm and a sequence $\left(U_{n \pi(n)}\right)$ of surjective isometries such that

$$
T\left(x_{n}\right)=\left(U_{n \pi(n)} x_{\pi(n)}\right)
$$

for each $\left(x_{n}\right) \in X$. Furthermore, $X_{\pi(n)}$ and $X_{n}$ are isometric for each $n$.

These conditions are also sufficient.

Proof. If $\pi$ is a symmetry of the sequence space norm and $\left(U_{n \pi(n)}\right)$ is a sequence of onto isometries then,

$$
\begin{aligned}
& \|T x\|=\left\|\left(\left\|(T x)_{n}\right\|\right)\right\|_{E}=\left\|\left(\left\|U_{n \pi(n)} x_{\pi(n)}\right\|_{n}\right)\right\|_{E}, \\
& \|T x\|=\left\|\left(\left\|x_{\pi(n)}\right\|_{\pi(n)}\right)\right\|_{E}=\left\|\left(\left\|x_{n}\right\|_{n}\right)\right\|_{E}=\|x\| .
\end{aligned}
$$

Thus the conditions are sufficient.

The necessity of the conditions given the hypothesis follows as in the proof of Theorem 2.5. The reason for this is that the hypotheses on $E$ imply by Theorem 3.3 that the hermitians are diagonal and the only other time that structure of $E$ enters in an essential way is that the norm on $E$ may not allow every possible permutation. This is where the symmetries of $E$ enter the picture.

Remark. If $E=l^{p}$ then an additional sufficient condition can be added, namely that each $X_{n}$ has trivial $L^{p}$ structure. The result is known and due to P. Greim [15].

Corollary 3.8. Let $\phi$ be an Orlicz function which is differentiable, satisfies the strong $\Delta_{2}$ property, and $t^{-1} \phi^{\prime}(t)$ is one to one. Let $\left(K_{n}\right)$ be a sequence of complex Hilbert spaces and $E=l_{\phi}$, the Orlicz sequence space associated with $\phi$. If $W=\oplus_{E} K_{n}$ and $T$ is a 
surjective isometry then there exist a symmetry $\pi$ of the E-norm and a sequence $\left(U_{k \pi(k)}\right)$ of unitary operators such that

$$
T\left(x_{n}\right)=\left(U_{n \pi(n)} x_{\pi(n)}\right) \text { for every } x=\left(x_{n}\right) \text { in } W .
$$

The converse is also true.

Remark. The hypothesis of Theorem 3.8 which is satisfied by the $K_{n}$ 's is (3.7d). This was observed by the authors in [9]. We know of no Banach space that satisfies (3.7d) which is not a Hilbert space. We suspect that this property may in fact characterize Hilbert space. The next proposition provides more evidence that our suspicions are correct.

Proposition 3.9. Let $X$ be a Banach space with a normalized 1-unconditional Schauder basis. If $A H(X) B=\{0\}$ implies that $A=0$ or $B=0$ then $X$ is a Hilbert space.

Proof. Let $\left(e_{i}\right)$ be a basis satisfying the hypothesis. Recall that two "coordinates" $i$ and $j$ are said to be equivalent if $\left|x_{i}\right|^{2}+\left|x_{j}\right|^{2}=\left|y_{i}\right|^{2}+\left|y_{j}\right|^{2}$ and $\left|x_{k}\right|=\left|y_{k}\right|$ for $k \neq i$ or $j$ implies $\left\|\sum x_{n} e_{n}\right\|=\left\|\sum y_{n} e_{n}\right\|$. To show that $X$ is Hilbert space it is sufficient to show that every pair of coordinates are equivalent.

In [8] we showed an operator $T$ is hermitian on $X$ if and only if its matrix $\left(t_{m n}\right)$ with respect to the basis $\left(e_{n}\right)$ satisfies the condition

$$
\begin{aligned}
& t_{m n}=0 \text { if } m \text { is not equivalent to } n \\
& t_{m n}=\bar{t}_{n m} \text { if } m \text { is equivalent to } n .
\end{aligned}
$$

Now suppose that for some pair of positive integers $i$ and $j$ that $i$ is not equivalent to $j$.

Define $A \in B(X)$ by $A=\left(a_{m n}\right)$ where

$$
\begin{array}{rrr}
a_{m n}=0 & \text { if } & (m, n) \neq(i, j) \\
1 & \text { if } & (m, n)=(i, j)
\end{array}
$$

Since $i$ is not equivalent to $j$, every hermitian operator $T$ has a matrix representation $\left(t_{m n}\right)$ in which $t_{i j}=t_{j i}=0$. It follows then that $A T A=0$ for every $T \in H(X)$ and this contradicts the hypothesis. Thus every pair of coordinates is equivalent and $X$ is a Hilbert space.

Remark. At this point we could give another application of the isometry theorem to some of the other spaces considered by LaDuke. Some of the spaces he considers are essentially $l^{p}$ sums of $B\left(H_{n}\right)$ 's where each $H_{n}$ is a finite dimensional Hilbert space. In this case each "factor space" satisfies $(3.7 \mathrm{~d})$. 
We should also point out that our theorem applies to the space $L_{[1]}$ of Schoenberg given in [17]. The space is an $l^{1}$ sum of spaces with trivial $L^{1}$ structure.

\section{Adjoint abelian operators on special sums}

The idea of an adjoint abelian operator was introduced in the paper of Stampfli [32].

Definition 4.1. $T \in B(X)$ is adjoint abelian (a.a.) if for some s.i.p. compatible with the norm on $X$ it is true that

$$
[T x, y]=[x, T y] \text { for every } x, y \in X .
$$

In general it seems that this class is quite small but Stampfli asked one question which still seems to be open. The question is whether or not a.a. operators must be scalar type spectral operators. We have answered this question $($ see $[10,11])$ for the special spaces, $C(\Omega)$, $\Omega$ compact metric, $L^{p}(\mu, H) 1<p<\infty, p \neq 2$, and $\oplus_{E} H_{k}$, when $H_{k}$ is a Hilbert space for each $k$ and $E=l^{p}$.

In this section we want to record some extensions of our earlier results.

The first is in keeping with the other sections of the paper but the second is in a slightly different setting than considered so far.

Proposition 4.2. Let $\left(X_{n}\right)$ be a sequence of Banach spaces and $E=l^{p}, 1<p<\infty, p \neq 2$. If $S=\oplus_{E} X_{n}$ then $A \in B(S)$ is adjoint abelian with respect to s.i.p.

$$
[x, y]=\sum\left[x_{k}, y_{k}\right]_{k}\left(\frac{\left\|y_{k}\right\|}{\|y\|}\right)^{p-2}
$$

if and only if

$$
A=\lambda U \text { where } U \text { is an isometry such that } U^{2}=I .
$$

Proof (outline). The converse is proven in [9]. Since $A^{2}=T$ is hermitian and adjoint abelian with respect to he same s.i.p. it follows that $T=\left(T_{k k}\right)$ and each $T_{k k}$ is a.a. on $X_{k}$. It follows from the definition and special choices of $x$ and $y$ in (4.2.1) that if $T_{k k} y_{k}=0$ for some $k$ then $y_{k}=0_{k}$. By an appropriate choice of $x$ and $y$ we can show that if $y_{k} \neq 0$ and $y_{j} \neq 0$ then

$$
\frac{\left\|T_{k k} y_{k}\right\|_{k}}{\left\|y_{k}\right\|}=\frac{\left\|T_{j j} y_{j}\right\|_{j}}{\left\|y_{j}\right\|}
$$

If we let $\lambda$ to be the common value then it follows that $\lambda^{-1} T$ is an isometry. From Theorem 6 of $[10]$ it follows that there exists an isometry $W$ such that $W^{2}=I$ and 
$T=\lambda W$. Now use the fact [33] that $\left\|A^{2}\right\|=\|A\|^{2}$ to get that $A$ is a multiple of an isometry $U$ and again by Theorem 6 of $[10], U^{2}=I$.

Remark. As we noted in [10], every a.a. operator satisfying the conclusion of the theorem is scalar type.

Given the previous result, it is natural to attempt to extend the proposition to the Bochner $L^{p}(\mu, X)$ spaces. At this time we can characterize a.a. operators on the Bochner $L^{p}$ spaces only for special $X$ 's and for finite measures. Thus we have:

Theorem 4.3. Let $L^{p}(\mu, X)$ be a Bochner space, $1<p<\infty, p \neq 2, \mu$ a probability measure and $X$ a Banach space with trivial hermitian operators. If $A$ is a.a. with respect to a s.i.p. [, ] on $L^{p}(\mu, X)$ for which

$$
[f, g]=\frac{1}{\|g\|^{p-2}} \int[f(t), g(t)]\|g(t)\|^{p-2} d \mu
$$

holds for simple functions $g \in L^{p}(\mu, X)$, then $A=\lambda U$ where $U$ is an isometry such that $U^{2}=I$.

Proof. Let $T=A^{2}$. Then $T$ is both hermitian and a.a. with respect to the given s.i.p. By Sourour's Theorem, [30], there exists a strongly measurable $H(X)$ valued function $K($.$) such that$

$$
(T f)(s)=K(s) f(s) \quad \text { for every } f \in L^{p}(\mu, X) .
$$

Since $H(X)=\{r I \mid r \in R\}$, there exists a bounded nonnegative scalar valued function $k(s)$ such that

$$
(T f)(s)=k(s) f(s) \quad \text { for every } \quad f \text { in } L^{p}(\mu, X)
$$

Furthermore, since $k(s)$ is nonnegative,

$$
[k(s) f(s), g(s)]=[f(s), k(s) g(s)]=k(s)[f(s), g(s)]
$$

for every $f$ and $g$ in $L^{p}(\mu, X)$.

Since $T$ is a.a. we have

$$
[T f, g]=[f, T g] \text { for every } f, g \text { in } L^{p}(\mu, X)
$$

and so for every simple $g$ we get

$$
\int k(s)[f(s), g(s)]\left(\frac{\|g(s)\|}{\|g\|}\right)^{p-2} d \mu=\int k(s)[f(s), g(s)]\left(\frac{\|k(s) g(s)\|}{\|T g\|}\right)^{p-2} d \mu
$$


Let $S(k)=\{s \mid k(s) \neq 0\}$ and $\sigma \subseteq S(k)$ with $\mu(\sigma)>0$. With $f=x_{\sigma} g$ we get from (4.3.6) that

$$
\int k(s) \frac{\|g(s)\|^{p}}{\|T g\|^{p-2}}\left(\left(\frac{\|T g\|}{\|g\|}\right)^{p-2}-k(s)^{p-2}\right) d \mu=0
$$

for every simple $g$. If we let $v \in X$ and set $g=v$ (the constant function) then $\|T g\|^{p}=\int|k(s) v|^{p} d \mu=\|k\|^{p}\|v\|^{p}$ and so from (4.3.7) we obtain

$$
\int \frac{k(s)\|v\|^{p}}{\|k\|^{p-2}\|v\|^{p}}\left[\|k\|^{p-2}-k(s)^{p-2}\right] d \mu=0 .
$$

From (4.3.8) we get that there exists $\sigma_{v} \subseteq s(k)$ with $\mu\left(\sigma_{v}\right)=0$ and $k(s)=\|k\|$ for all $s \in S(k) \backslash \sigma_{v}$.

We claim that $\mu(\Omega \backslash S(k))=0$. For suppose that $\mu(\Omega \backslash S(k))>0$. Let $\delta_{1} \subseteq \Omega \backslash S(k)$ and $\delta_{2} \subseteq S(k)$ be sets of positive measure. Let $v_{1}, v_{2}$ be in $X$ and set $y=\chi_{\delta_{1}} v_{1}+\chi_{\delta_{2}} v_{2}$. If $\left\|v_{1}\right\|=\left\|v_{2}\right\|=1$ then $\|g\|^{p}=\mu\left(\delta_{1}\right)+\mu\left(\delta_{2}\right)$, and

$$
(T g)(s)=k(s) \chi_{\delta_{1}}(s) v_{1}+k(s) \chi_{\delta_{2}}(s) v_{2}=k(s) \chi_{\delta_{2}}(s) v_{2} .
$$

Thus, $\|T g\|^{p}=\|k\|^{p} \mu\left(\delta_{2}\right)$, and

$$
\frac{\|T g\|^{p-2}}{\|g\|^{p-2}}=\frac{\|k\|^{p-2} \mu\left(\delta_{2}\right)^{r}}{\left(\mu\left(\delta_{1}\right)+\mu\left(\delta_{2}\right)\right)^{r}} \quad \text { where } \quad r=(p-2) / p
$$

If we use (4.3.9) in (4.3.7) with $\sigma=\delta_{2}$ and the fact that $k(s)=\|k\|$ we obtain:

$$
\int \frac{\|k\|}{\mu\left(\delta_{2}\right)^{r}}\left[\frac{\mu\left(\delta_{2}\right)^{r}}{\left(\mu\left(\delta_{1}\right)+\mu\left(\delta_{2}\right)\right)^{r}}-1\right] d \mu=0
$$

This gives us

$$
\frac{\|k\|}{\mu\left(\delta_{2}\right)^{r}} \mu\left(\delta_{2}\right)\left[\frac{\mu\left(\delta_{2}\right)^{r}}{\left(\mu\left(\delta_{1}\right)+\mu\left(\delta_{2}\right)\right)^{r}}-1\right]=0 .
$$

This last equation is not possible if $\mu\left(\delta_{1}\right)>0$. Therefore, for every measurable subset $\delta_{2} \subseteq \Omega \backslash S(k), \mu\left(\delta_{2}\right)=0$. We conclude that $k(s)=\|k\|$ a.e.

We have just shown that $A^{2}=\|k\| I$ and we can conclude that $A=\|k\| W$ for some isometry $W$ for which $W^{2}=1$.

Remarks. First of all if $X$ is smooth then $L^{p}(\mu, X)$ has a unique s.i.p. $(1<p<\infty)$, and formula (4.3.1) holds for every $f$ and $g$ in $L^{p}(\mu, X)$. Furthermore smooth spaces with trivial hermitians exist, e.g. see [12].

This theorem raises several questions 
(4.Q.1) Does the result of Theorem 4.3 hold for $X$ 's with nontrivial hermitians?

(4.Q.2) Which Banach spaces $X$ can be equivalently renormed so that $H(X)$ is trivial? In particular what happens if $X$ is smooth?

(4.Q.3) Does the result of Theorem 4.3 hold for $C(\Omega, X)$ when $\Omega$ is compact metric?

(4.Q.4) Is every a.a. operator on $C_{p}(H)$, the Schatten $p$-class, $1<p<\infty, p \neq 2$, a scalar operator?

\section{Hyponormal operators-some special cases}

K. Mattila [23] introduced the notion of a hyponormal operator on a Banach space $X$.

Definition 5.1. $T \in B(X)$ is hyponormal iff there exist hermitian operators $H$ and $K$ such that $T=H+i K$ and $i(H K-K H) \geqq 0$, i.e. the numerical range of $i(H K-K H)$ is contained in $\mathbb{R}^{+}$.

The idea of a normal operator was defined earlier by Lumer. He defined an operator $T \in B(X)$ to be normal if $T=H+i K$ where $H$ and $K$ are hermitian operators such that $H K-K H=0$.

Remarks. Given the characterizations of the hermitians on the spaces in question, it is obvious that on the spaces $L^{p}(\mu$,$) , reflexive Orlicz spaces L_{\phi}(\mu)$ and $C(\Omega)$ for $\Omega$ compact, the hyponormal operators and the normal operators are the same class.

Proposition 5.2. Let $Y=L^{p}(\mu, X) 1 \leqq p<\infty, p \neq 2$, or $C(\Omega, X)$, $\Omega$ compact Hausdorff, or $Y=\oplus_{E} X_{n}$, where $E$ is admissible. $T$ is hyponormal on $Y$ iff there exists an operator valued function $A(t)$ in $B(X)\left(A(n) \in B\left(X_{n}\right)\right.$ in the last case) such that $A(t)$ is hyponormal on $X$ (or $X_{n}$ in the last case) for which

$$
(T f)(s)=A(s) f(s) \quad \text { for every } f \in Y \text {. }
$$

Proof. The proofs of all cases are essentially the same so we will give only the proof for $C(\Omega, X)$. Let $T=K_{1}+i K_{2}$ where $K_{1}, K_{2}$ are hermitian and $i\left(K_{1} K_{2}-K_{2} K_{1}\right) \geqq 0$. From [13] it is clear that there exists $H(X)$ valued functions $K_{1}(s)$ and $K_{2}(s)$ such that $T f(s)=\left(K_{1}(s)+i K_{2}(s)\right) f(s)$ for every $s \in \Omega$. Let $\delta(s)=i\left(K_{1}(s) K_{2}(s)-K_{2}(s) K_{1}(s)\right)$. We need to show that $\delta(s) \geqq 0$ for each $s \in \Omega$. To do this let $[$,$] be any compatible s.i.p. for X$. Let $\psi$ be a choice function with domain $C(\Omega, X)$ and range $\Omega$ such that $\psi(g) \in\{s \mid\|g(s)\|=\|g\|\}$. Then

$$
[f, g]_{\psi}:=[f(\psi(g)), g(\psi(g))]
$$

is a s.i.p. which is compatible with the norm on $C(\Omega, X)$. 
If $x \in X$, let $\mathbf{x}$ denote the constant function which takes the value $x$ at every point. Since $T$ is hyponormal

$$
[\delta \mathbf{x}, \mathbf{x}]_{\psi}=\left[i\left(K_{1}(\psi(x)) K_{2}(\psi(x))-K_{2}(\psi(x)) K_{1}(\psi(x))\right) x, x\right] \geqq 0 .
$$

Since $\|\mathbf{x}(s)\|=\|\mathbf{x}\|$ for every $s \in \Omega$, and since each choice function leads to a compatible s.i.p. on $C(\Omega, X)$, it follows that the operator $i\left(K_{1}(s) K_{2}(s)-K_{2}(s) K_{1}(s)\right) \geqq 0$ for each $s \in \Omega$.

In ([23, Theorem 2.4]) Mattila show that if $T=H+i K$ is hyponormal on $X$ and $X$ is complex strictly convex then $T x=0$ iff $H x=K x=0$. Using this result and Proposition 5.2 we have:

Corollary 5.3. Let $X$ be complex strictly convex and $\Omega$ compact Hausdorff. If $T$ is a hyponormal operator on $C(\Omega, X)$ and $T f=H f+i K f=0$ then $H f=K f=0$.

Proof. Suppose $T=H+i K$ is hyponormal and $T f=0$. By Proposition 5.2, there exists an operator valued functions $K_{j}(s)$ such that $K_{j}(s)$ is hermitian for each $s \in \Omega$ and $K_{1}(s)+i K_{2}$ is hyponormal on $X$ for each $s$. So $T f=0$ implies that $T f(s)=K_{1}(s) f(s)+$ $i K_{2}(s) f(s)=0$. It follows by Mattila's theorem that $K_{1}(s) f(s)=0$ and $K_{2}(s) f(s)=0$ for each $s$. Thus $H f=0$ and $K f=0$.

Remark. This corollary shows that complex strict convexity of the range space in Mattila's Theorem 2.4 is not a necessary condition because $C(\Omega, X)$ is not complex strictly convex if $\Omega$ contains at least two points.

In Hilbert space it is known that of an operator is both compact and hyponormal then it is normal. So it is a natural question to ask, is every compact hyponormal operator on a Banach space normal? This result is true for Banach spaces with 1unconditional bases. The reason for this is that the hermitians are diagonal and hence hyponormal operators are diagonal. Hyponormality of the original operator implies that the diagonal elements are hyponormal on their domains. Compactness of the operator implies compactness of the diagonal operators and since the factor spaces are Hilbert spaces the diagonal operators are normal and consequently the original operator is normal. This type of argument can be extended to a different setting as follows:

Proposition 5.4. Let $T$ be a hyponormal operator on $C(\Omega, X)$ where $X$ is a Hilbert space. If $T$ is compact and hyponormal then $T$ is normal.

Proof. We have already shown that there exists a function $A(s)$ defined on $\Omega$ with values in $B(X)$ such that $A(s)$ is hyponormal for every $s$ and $(T f)(s)=A(s) f(s)$. To prove that $T$ is normal it is sufficient to prove that $A(s)$ is compact for each $s$.

Let $\left(x_{n}\right)$ be a bounded sequence in $X$. The sequence of constant functions $\left(\mathbf{x}_{n}\right)$ is bounded in $C(\Omega, X)$. Since $T$ is compact there exists a subsequence $x_{n}$ such that $T \mathbf{x}_{n_{k}}$ is convergent to some $g \in C(\Omega, X)$. So 


$$
\left\|T \mathbf{x}_{n_{k}}-g\right\| \rightarrow 0 \text { as } k \rightarrow \infty
$$

Hence

$$
\left\|T x_{n_{k}}(s)-g(s)\right\| \rightarrow 0 \text { uniformly in } s
$$

Thus,

$$
\left\|A(s) x_{n k}-g(s)\right\| \rightarrow 0 \text { uniformly in } s .
$$

It follows that for each $s, A(s)$ is compact. Since $A(s)$ is also a hyponormal and $X$ is a Hilbert space, $A(s)$ is normal for each $s$.

\section{Banach spaces with trivial hermitians and the Banach Stone Theorem}

In this section we will use property (2.3c) of Lemma 2.3 plus a result from [13] to get a Banach Stone type Theorem. Before proceeding with this we note that Behrends has a stronger result [4], and we are just offering a different proof for the complex case. Before stating the theorem we recall that a Banach space is said to have trivial hermitians if the only hermitian operators are real multiples of the identity.

Proposition 6.1. Let $\Omega_{1}$ and $\Omega_{2}$ be compact Hausdorff spaces and $X_{1}, X_{2}$ Banach spaces with trivial hermitians. An operator $T: C\left(\Omega_{1}, X_{1}\right) \rightarrow C\left(\Omega_{2}, X_{2}\right)$ is a surjective isometry iff there exists a homeomorphism $\tau: \Omega_{2} \rightarrow \Omega_{1}$ and a surjective isometry $U: X_{1} \rightarrow X_{2}$ such that

$$
(T f)(w)=U(w) f(\tau(w)) \quad \text { for every } \quad f \in C\left(\Omega_{1}, X_{1}\right) .
$$

Proof. Let $h \in C_{R}\left(\Omega_{1}\right)$. Then $\left(M_{h} f\right)(w)=h(w) f(w)$ clearly defines a hermitian operator on $C\left(\Omega_{1}, X_{1}\right)$. By Lemma $2.3 T M_{h} T^{-1}$ is hermitian on $C\left(\Omega_{2}, X_{2}\right)$. From Theorem 4 of [13] and the hypothesis that $X_{2}$ has only trivial hermitians it follows that there exists a unique $g \in C_{R}\left(\Omega_{2}\right)$ such that

$$
T M_{h} T^{-1}=M_{g} .
$$

Define $\phi(h)=g$. It follows easily that $\phi$ is an algebra isomorphism of $C_{R}\left(\Omega_{1}\right)$ into $C_{R}\left(\Omega_{2}\right)$. To see that $\phi$ is onto, let $g \in C_{R}\left(\Omega_{2}\right)$. Then $M_{g}$ is hermitian on $C\left(\Omega_{2}, X_{2}\right)$. By Lemma $2.3 T^{-1} M_{g} T$ is hermitian on $C\left(\Omega_{1}, X_{1}\right)$. Hence there is a $k \in C_{R}\left(\Omega_{1}\right)$ with $T^{-1} M_{g} T=M_{k}$. Clearly $\phi(k)=g$ and so $\phi$ is a algebra isomorphism of $C_{R}\left(\Omega_{1}\right)$ onto $C_{R}\left(\Omega_{2}\right)$. Again from [13] we obtain a homeomorphism $\tau: \Omega_{2} \rightarrow \Omega_{1}$ such that

$$
\phi(h)=h o \tau \text {. }
$$

For each $w \in \Omega_{1}$ and $x \in X_{1}$, define 


$$
U(w) x=T \mathbf{x}(w) \text {, where } \mathbf{x}(w)=x \text { for every } w \in \Omega_{1} .
$$

$U(w)$ is clearly a linear transformation from $X_{1}$ to $X_{2}$. Furthermore, $U(w)$ is uniformly bounded since

$$
\|U(w) x\|=\|T \mathbf{x}(w)\| \leqq\|T \mathbf{x}\|_{\infty}=\|\mathbf{x}\|_{\infty}=\|x\| .
$$

We claim that not only is $U(w)$ uniformly bounded but in fact $U(w)$ is an isometry for each $w$. For suppose there is an $x_{0} \in X_{1}$ of norm 1 and $w_{0}$ such that $\left\|T x_{0}\left(w_{0}\right)\right\|<1$. Then there is a neighbourhood $0_{1}$ of $w$ with $\left\|x_{0}(w)\right\|<1$ for all $w \in 0_{1}$. Since $\Omega_{2}$ is normal, there is an $0_{2}$ such that $0_{2}^{-} \subseteq 0_{1}$. By Urysohn's Lemma there exists an $f \in C_{R}\left(\Omega_{2}\right)$ such that $f\left(\Omega_{2}\right) \subseteq[0,1]$ and $f\left(\overline{0}_{2}\right)=1$ and $f\left(0_{1}^{c}\right)=0$. Let $g:=\left(f 0 \tau^{-1}\right) x_{0}$. Then $g$ belongs to $C\left(\Omega, X_{1}\right.$ and $\|g\|=1$. However,

$$
\begin{gathered}
T g(w)=T M_{g} T^{-1} T x_{0}(w)=f(w) T x_{0}(w) \quad \text { and so } \\
\|T g\|_{\infty}=\operatorname{Max}_{w} \mid f(w)\|\| T \mathbf{x}_{0}(w) \|<1
\end{gathered}
$$

and this contradicts the fact $T$ is an isometry. Therefore

$$
\|U(w) x\|=\|x\| \text { for every } x \in X .
$$

We have shown that for $f \in C_{R}\left(\Omega_{1}\right), \quad x \in X_{1}$

$$
\begin{gathered}
T(f x)(w)=T M_{f} T^{-1} \mathbf{x}(w)=f(\tau(w)) U(w) x \quad \text { or } \\
T(f x)(w)=U(w) f(\tau(w)) x .
\end{gathered}
$$

Since linear combinations of functions of the form $f(w) x$ are dense in $C\left(\Omega_{1}, X_{1}\right)$ we have shown that (6.1.1) holds if $T$ is a surjective isometry of $C\left(\Omega_{1}, X_{1}\right)$ onto $C\left(\Omega_{2}, X_{2}\right)$. The converse is obvious.

Remarks. We wish to point out that because of Lemma 2.3 most of the isometry theorems in this paper could be stated in a slightly more general way. For example, Theorem 3.7 could be stated for an isometry between an $E$ sum of $\left\{X_{i}\right\}$ and an $E$ sum of $\left\{Y_{i}\right\}$ provided the $X_{i}$ satisfied the hypotheses of (3.7).

\section{REFERENCES}

1. J. Arazy, Isometries of complex symmetric sequences spaces, Math. Z. 188 (1985), 317-321.

2. S. BANach, Theorie des operations lineaires (Chelsea, New York, 1955).

3. E. Behrends, et al., $L^{p}$ structure in real Banach spaces (Springer Lecture Notes in Math. 613, 1977). 
4. E. Behrends, M-structure and the Banach Stone theorem (Springer Lecture Notes in Math. 736, 1979).

5. E. Berkson and A. Sourour, The hermitian operators on some Banach spaces, Studia Math. 52 (1974), 33-41.

6. D. Borwein, Linear functionals connected with strong Cesaro summability, J. London Math. Soc. 40 (1965), 628-634.

7. M. M. DAY, Reflexive Banach spaces not isomorphic to uniformly convex spaces, Bull. Amer. Math. Soc. 47 (1941), 313-317.

8. R. J. Fleming and J. E. JAMISON, Hermitian and adjoint abelian operators on certain Banach spaces, Pacific J. Math. 52 (1974), 67-84.

9. R. J. Fleming and J. E. Jamison, Isometries on certain Banach spaces, J. London Math. Soc. 9 (1975), 121-127.

10. R. J. Fleming and J. E. Jamison, Adjoint abelian operators on $L^{p}$ on $C(K)$, Trans. Amer. Math. Soc. 217 (1976), 87-98.

11. R. J. Fleming and J. E. Jamison, Classes of operators on vector valued integration spaces, J. Austral. Math. Soc. Ser. A 24 (1977), 128-129.

12. R. J. Fleming and J. E. Jamison, The Isometries of Sp( $\alpha$ ), Canad. J. Math. 33 (1981), 59-67.

13. R. J. Fleming and J. E. Jamison, Hermitian operators on $C(X, E)$ and the Banach-Stone theorem, Math. Z. 170 (1980), 77-84.

14. R. J. Fleming, Jerome Goldstein and J.E. Jamison, One parameter groups of Isometries on certain Banach Spaces, Pacific J. Math. 64 (1976), 145-151.

15. P. Greim, Isometries and $L^{p}$-strucure of separably valued $L^{p}$-spaces, in: Measure Theory and Its Applications, Proc. Conf. Sherbrooke 1982 (Springer Lecture Notes in Math. 1033, Berlin, Heidelberg, New York, 1983), 209-218.

16. P. Greim, Hilbert spaces have the Banach-Stone property for Bochner spaces, Bull. Austral. Math. Soc. 27 (1983), 121-128.

17. A. Jakimovskı and D. C. Russell, Representation of continuous linear functionals on a subspace of a continuous linear functionals on a subspace of a countable cartesian product of Banach spaces, Studia Math. 72 (1982), 274-284.

18. J. Jamison, Irene Loomis and C. C. Rousseau, Complex convexity of certain Banach spaces, MH. Math. 99 (1985), 199-211.

19. J. LADUKE, On a certain Generalization of lp spaces, Pacific J. Math. 35 (1970), 155-168.

20. J. Lamperti, On Isometries of certain function spaces, Pacific J. Math. 2 (1985), 459-466.

21. G. Lumer, Semi inner product spaces, Trans. Amer. Math. Soc. 100 (1961), 29-43.

22. G. Lumer, On the Isometries of reflexive Orlicz spaces, Ann. Inst. Fourier (Grenoble) 13 (1963), 99-109. 
23. K. Matrila, Complex strict and uniform convexity and hyponormal operators, Math. Proc. Cambridge, Philos. Soc. 96 (1984), 483-493.

24. J. R. Partington, Hermitian operators for absolute norms and absolute direct sums, Linear Algebra Appl. 23 (1979), 275-280.

25. R. PAYA'-Albert, Numerical range of operators and structure of Banach Spaces, Quart. $J$. Math. Oxford (2), 33 (1982), 357.

26. H. Rosenthal, Functional Hilbert sums, (1985), preprint.

27. H. RosenthaL, The Lie algebra of Banach space, (1985), preprint.

28. H. Schneider and R. E. L. Turner, matrices hermitian for an absolute norm, Linear and Multilinear Algebra 1 (1973), 9-32.

29. A. M. SinClair, Jordan homomorphism and derivations on semi simple Banach algebras, Proc. Amer. Math. Soc. 24 (1970), 209-214.

30. A. R. Sourour, Isometries of $L^{p}(\mu, X), J$. Funct. Anal. 30 (1978), 276-285.

31. A. R. Sourour, Isometries of norm ideals of compact operators, J. Funt. Anal. 43 (1981), 69-77.

32. J. G. Stamprli, Adjoint abelian operators on Banach space, Canad. J. Math. 21 (1969), 505-512.

33. D. van Dulst and V. DeValk, (KK)-Properties, Normal Structure and fixed points on nonexpansive mappings in Orlicz sequence spaces, Canad. J. Math. 38 (1986), 728-750.

Department of Mathematics

Central Michigan University

Mount Pleasant, Michigan

U.S.A.
Department of Mathematics Memphis State University Memphis, TN. 38152

U.S.A. 